\title{
Engineering multi-specific antibodies against HIV-1
}

\author{
Neal N. Padte, Jian Yu, Yaoxing Huang and David D. Ho*
}

\begin{abstract}
As increasing numbers of broadly neutralizing monoclonal antibodies (mAbs) against HIV-1 enter clinical trials, it is becoming evident that combinations of mAbs are necessary to block infection by the diverse array of globally circulating HIV-1 strains and to limit the emergence of resistant viruses. Multi-specific antibodies, in which two or more HIV-1 entry-targeting moieties are engineered into a single molecule, have expanded rapidly in recent years and offer an attractive solution that can improve neutralization breadth and erect a higher barrier against viral resistance. In some unique cases, multi-specific HIV-1 antibodies have demonstrated vastly improved antiviral potency due to increased avidity or enhanced spatiotemporal functional activity. This review will describe the recent advancements in the HIV-1 field in engineering monoclonal, bispecific and trispecific antibodies with enhanced breadth and potency against HIV-1. A case study will also be presented as an example of the developmental challenges these multi-specific antibodies may face on their path to the clinic. The tremendous potential of multi-specific antibodies against the HIV-1 epidemic is readily evident. Creativity in their discovery and engineering, and acumen during their development, will be the true determinant of their success in reducing HIV-1 infection and disease.
\end{abstract}

Keywords: HIV-1, Bispecific antibody, Trispecific antibody, Multi-specific antibody, Neutralizing antibody, Passive immunization

\section{Background}

The past decade has introduced a new generation of potent and broad neutralizing monoclonal antibodies (mAbs) against HIV-1 [1-10], several of which have entered the clinic recently [11-17]. This resurgence of promising HIV-1 mAbs has energized the field of passive immunization and propelled the testing of existing mAbs as treatment, particularly because of their long half-lives as compared to existing oral antiretroviral options. The high degree of HIV-1 envelope (Env) diversity, however, requires further improvements to these mAbs to better ensure their clinical utility. For example, viral resistance can rapidly evade antiviral pressure from a single $\mathrm{mAb}$ treatment $[11,12,14,18,19]$, and a large fraction of circulating HIV-1 already exhibit pre-existing resistance to many of the antibodies currently in development [20-22].

*Correspondence: dho@adarc.org

Aaron Diamond AIDS Research Center, The Rockefeller University, 455 First Avenue, New York, NY 10016, USA
HIV-1 mAbs directed to more conserved components of the viral entry process, such as ibalizumab, which binds to the CD4 receptor on T-cells [23], and PRO140, which binds to the CCR5 co-receptor [24], broadly neutralize a greater fraction of circulating HIV-1 than Envtargeting mAbs $[20,25]$. Indeed, ibalizumab $\left(\operatorname{Trogarzo}^{\circledR}\right)$ has recently become the first mAb against HIV-1 to receive FDA approval and is currently indicated for use as salvage therapy in patients whose viruses are resistant to multiple existing antiretroviral drugs [26, 27]. PRO140 is currently in a Phase $2 b / 3$ pivotal trial in heavily treatment-experienced HIV-1 patients [28]. However, these promising antibodies must be used in combination with other antiretroviral agents to limit emerging viral resistance. While the newer generation of Env-targeting mAbs that have recently entered Phase 1 trials are more potent and broad than earlier generations of HIV-1 Env-targeting $\mathrm{mAbs}$, they still face these same issues of viral resistance unless they can be administered in combinations, and this costly undertaking could limit their practical feasibility, particularly in the setting of HIV-1 prevention in 
under-resourced nations [29]. Engineering antibodies for greater HIV-1 neutralization and breadth, particular by the creation of bispecific and trispecific antibodies, and for improved in vitro stability and in vivo pharmacokinetics, has the potential to drastically reduce the amount of antibody required for efficacy in humans, and may put the goal of an efficacious HIV-1 prevention and therapeutic antibody strategy within reach.

\section{Engineering $m A$ abs to improve potency and breadth against HIV-1}

One strategy to improve HIV-1 mAbs is to use structureguided design to develop rationally engineered antibody variants with improved antiviral properties. Many of the engineering principles applied to these HIV-1 mAbs were also incorporated into the investigational studies to engineer multi-specific antibodies reviewed in this article, and therefore a short summary of these structure-guided engineering approaches for HIV-1 mAbs will be reviewed first.

\section{Engineering $\mathrm{CD} 4$ binding site $\mathrm{mAbs}$}

The HIV-1 CD4 binding site antibody NIH45-46 was identified as a more potent clonal variant of VRC01 [6, 10]. Structural studies determined that NIH45-46 lacked a critical interaction to a hydrophobic pocket between the gp120 bridging sheet and outer domain that is typically occupied by a phenylalanine on CD4, and it was reasoned that a hydrophobic residue at position 54 on NIH45-46 could improve its interaction with gp120. After engineering one of a series of hydrophobic residues at this position 54 , the variant $\mathrm{NIH} 45-46^{\mathrm{G} 54 \mathrm{~W}}$ was found to increase contact with the gp120 bridging sheet and improved its neutralization potency by tenfold [30].

VRC07, another somatic variant of VRC01, was engineered with improved binding to the HIV-1 CD4 binding site by incorporating a histidine mutation at the G54 position of this antibody (the same position as that mutated in $\mathrm{NIH} 45-46^{\mathrm{G} 54 \mathrm{~W}}$ ). VRC07 was also engineered with several mutations in its light chain to increase solubility and to remove a potential N-linked glycosylation site, which together resulted in a 7.9-fold enhancement in potency as compared to VRC01 and with reduced autoreactivity as compared to $\mathrm{NIH} 45-46^{\mathrm{G} 54 \mathrm{~W}}$ [5]. A variant of VRC07-523 engineered to have a longer half-life in vivo (VRC07-523-LS) demonstrated protective efficacy at one-fifth of the dose of VRC01-LS in a non-human primate model, and is currently in Phase 1 clinical evaluation [16].

\section{Engineering MPER binding site $\mathrm{mAbs}$}

A similar approach to improve antibody solubility and potency was taken for the gp41 membrane proximal external region (MPER) binding antibody, 10E8 [3]. 10E8 was identified from an HIV-1 infected individual and is one of the broadest antibodies reported to date, neutralizing $>95 \%$ of circulating HIV-1 strains. However, $10 \mathrm{E} 8$ is naturally prone to aggregation, which limited its clinical manufacturability potential. By identifying somatic variants of $10 \mathrm{E} 8$ with inherently better solubility, and then using structural data to mutate a hydrophobic patch distal from the binding site of this antibody, a significantly more soluble variant of 10E 8 was obtained [31]. Because germline variants often exhibit reduced potency compared to their affinity matured antibody counterparts, residues from 10E8 critical for binding to MPER were then grafted onto this more soluble antibody. The new 10E8 variants retained the improved solubility but now also exhibited potency similar to the originally identified 10E8. The top variants, 10E8v4 and 10E8v5, exhibited improved pharmacokinetic profiles in mice and rhesus macaques as compared to 10E8, and 10E8v5 has been advanced for clinical evaluation [32]. An additional $10 \mathrm{E} 8 \mathrm{v} 4$ variant, known as $10 \mathrm{E} 8 \mathrm{v} 4-5 \mathrm{R}+100 \mathrm{cF}$, was recently reported to improve the potency of $10 \mathrm{E} 8 \mathrm{v} 4$ by an additional $\sim 10$-fold using a surface-matrix screening approach [33].

\section{Engineering a CD4-targeting $\mathrm{mAb}$}

In addition to engineering antibodies for improved solubility and potency against HIV-1, improved breadth of neutralization against circulating HIV-1 strains has also been demonstrated, which has the potential to erect a higher genetic barrier to viral resistance. The aforementioned CD4-targeting antibody, ibalizumab, already demonstrated favorable potency and breadth against circulating HIV-1 strains [20]. It neutralized $92 \%$ of viruses tested in vitro as assessed by $\geq 50 \%$ neutralization, but only neutralized $66 \%$ of viruses when assessed as $\geq 80 \%$ inhibition. This indicated that a significant fraction of circulating viruses may be able to escape complete neutralization. These studies revealed a strong correlation between HIV-1 resistance to ibalizumab and a loss of a V5 glycan on the viral envelope. In a separate study in HIV-1 infected patients in which ibalizumab monotherapy was added to failing drug regimens, a transient decrease in viral load was followed by evolution of resistant HIV-1 variants with a similar loss of a V5 glycosylation site [19]. Taken together with epitope mapping and X-ray crystallography structural studies used to define the ibalizumabCD4 binding interface $[34,35]$, it was hypothesized that the loss of the HIV-1 V5 glycan provided the viral envelope more flexibility to circumvent the steric hindrance induced by ibalizumab. To address this deficiency in ibalizumab, a panel of variants was engineered with glycans added to the ibalizumab light chain at positions predicted 
to sterically fill the empty space created by the loss of V5 glycan in the resistant viruses [36]. These modified glycan variants were able to neutralize HIV-1 strains previously resistant to ibalizumab, and the top variant, known as LM52, neutralized $100 \%$ of circulating HIV-1 strains tested as assessed by $\geq 80 \%$ neutralization, and at a potency $\sim 5$ - to 10-fold better than wild-type ibalizumab. LM52 is currently in preclinical development in preparation for clinical evaluation [37].

The examples presented above demonstrate how structure-guided approaches and rational design, in combination with germline antibody identification, can improve the potency, breadth and solubility of multiple antibodies against HIV-1, and several of these are currently in preclinical or clinical development. However, even with these improvements, the dynamics of HIV-1 viral replication and the rapid mutation rate of HIV-1 require these antibodies be used in combinations in order to limit the emergence of resistant viruses in a treatment setting and in order to block infection by a diverse range of circulating subtypes in a prevention setting. While such combinations of antibodies are currently being explored [22], the high cost of development and delivery of these biologic combinations has the potential to limit their widespread use, necessitating alternative solutions.

\section{Engineering multi-specific antibodies to improve breadth against HIV-1}

The idea that multi-specific antibodies could improve upon the functional activities of single mAbs or combinations of mAbs originated in the cancer therapy field in the mid-1980s, primarily as a way to direct effector cells toward tumor cells [38-40]. As a result, the majority of bispecific antibodies currently under clinical evaluation today are for the treatment of various cancers [41]. The need for multi-specific antibodies for HIV-1 prevention and treatment, however, is readily evident. Multiple HIV-1 targeting epitopes can be incorporated into one antibody-like molecule, allowing for increased neutralization breadth against diverse HIV-1 strains and thereby also erecting a higher genetic barrier for viral resistance. Additionally, the large array of multi-specific antibody formats currently available [42] allow the tailoring of any particular combination of HIV-1 targeting antibody moieties by a number of structural properties such as size, distance, and valency in order to meet the requirements of viral inhibition.

\section{Engineering bispecific antibodies with improved breadth}

One example of a bispecific antibody that can enhance neutralization breadth is iMabm36 [43], which inhibits HIV-1 entry by targeting CD4, via ibalizumab (iMab), and the gp120 co-receptor binding site, via the antibody domain $\mathrm{m} 36$. This bispecific antibody is generated by genetically linking $\mathrm{m} 36$ to the $\mathrm{C}$-terminus of the ibalizumab heavy chain (Fig. 1a). As stated earlier, ibalizumab neutralizes $66 \%$ of viruses when assessed as $\geq 80 \%$ inhibition, indicating a significant fraction of circulating viruses may escape complete neutralization by ibalizumab. In contrast, the bispecific antibody iMabm36 neutralized $87 \%$ of viruses as defined by $\geq 80 \%$ inhibition, indicating a substantial improvement in neutralization breadth. This is attributed to the presence of two distinct HIV-1 entry inhibiting antibody domains within the same molecule. Improved antiviral activity was dependent on both the CD4-binding activity of the iMab component as well as the gp120 coreceptor-binding activity of the m36 component, as knocking out the activity of either of these components within the iMab36 molecule greatly reduced its antiviral activity. The linker length between the m36 antibody domain and the C-terminus of the iMab heavy chain also affected the antiviral activity of the bispecific antibody, suggesting that the flexibility and position of the fused domains relative to one another are also important for the functional activity of iMabm36.

In a separate line of study, a panel of bispecific antibodies was engineered in which one of several gp120-targeting single-chain variable fragments $(\mathrm{scFv})$ was fused to the $\mathrm{N}$-terminus or $\mathrm{C}$-terminus of the ibalizumab heavy chain (Fig. 1b, c) [44]. A number of variations of this format were also engineered, including those which inverted the orientation of variable domains within the $\mathrm{scFv}$ (for example, $V_{H}$ followed by $V_{L}$, or $V_{L}$ followed by $V_{H}$ ), and those which varied the linker lengths between the $V_{H}$ and $\mathrm{V}_{\mathrm{L}}$ domains within each scFv or between the $\mathrm{scFv}$ domains and the ibalizumab heavy chain. Interestingly, the binding and neutralization activity of each of these bispecific antibody variants varied widely, and the most optimal format in terms of $\mathrm{V}_{\mathrm{H}}$ and $\mathrm{V}_{\mathrm{L}}$ orientation and linker lengths differed depending on which HIV-1 Envtargeting $\mathrm{scFv}$ was fused to ibalizumab. Therefore, identifying an optimal bispecific antibody format and design, even within the context of structure-guided rational design of HIV-1 antibody-epitope pairings, is still an empirical process.

Ibalizumab fused to gp120 CD4 binding site antibodies, such as VRC01, NIH45-46 $6^{\mathrm{G} 4 \mathrm{~W}}$, or 3BNC60, neutralized $>99 \%$ of circulating HIV-1 strains tested, as assessed by $\geq 50 \%$ neutralization, and with a geometric mean $\mathrm{IC}_{50}$ ranging from 0.025 to $0.031 \mu \mathrm{g} / \mathrm{mL}$. These bispecific antibodies also neutralized $>97 \%$ of strains tested, as assessed by $\geq 80 \%$ inhibition, with a geometric mean $\mathrm{IC}_{80}$ ranging from 0.076 to $0.092 \mu \mathrm{g} / \mathrm{mL}$. This significant enhancement in neutralization breadth when ibalizumab was fused to each of these gp120 CD4 binding site antibodies indicates that these sets of parental antibody pairings could prove 


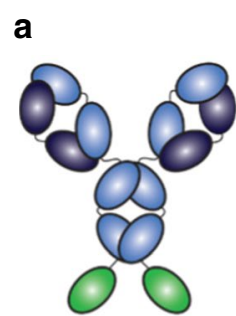

iMabm36

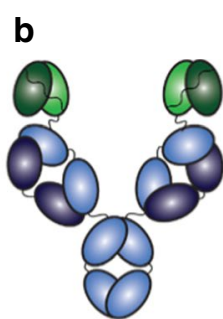

3BNC60-iMab PG9-iMab
C

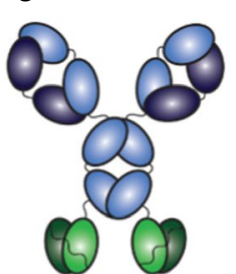

iMab-VRC01 d

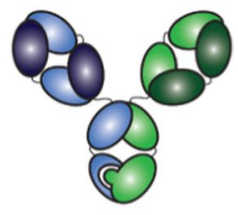

VRC07-PG9-16

10E8.4/iMab* e

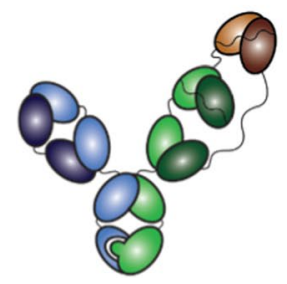

VRC01/PGDM 1400-10E8v4* f

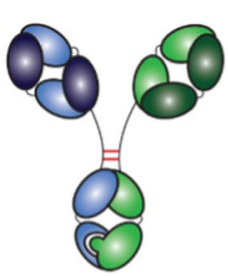

3BNC117/ g

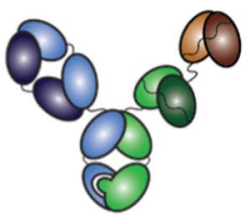

10E8v4/PGT h

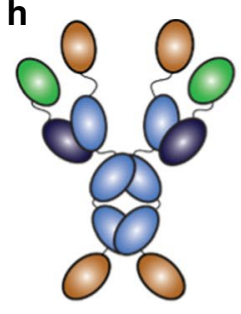

4Dm2m i

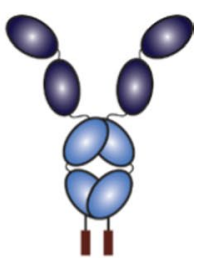

eCD4-Ig*

Fig. 1 Multi-specific antibody formats engineered for the prevention and treatment of HIV-1. a lgG-Fv fusion, b, c lgG-scFv, d CrossMAb, e $\mathrm{KiH}-\mathrm{CODV}-\mathrm{lgG}, \mathbf{f}$ IgG3C-, g KiH + tandem scFvs, h tetravalent + bivalent Fc-fusion, i Fc-fusion peptide. Representative multi-specific antibodies are listed under their respective schematic. *Means currently in clinical development

optimal in neutralizing a diverse sequence of circulating HIV-1 strains. Indeed, as mentioned earlier, a strong correlation was observed between HIV-1 resistance to ibalizumab and a loss of a V5 glycan on the viral envelope [20] and, in contrast, resistance to VRC01 involves the presence of bulky V5 residues [45]. Therefore, by combining two antibodies with complimentary resistance profiles into a single bispecific antibody, tremendous enhancements in neutralization breadth at or close to $100 \%$ can be achieved.

The CrossMAb format for engineering bispecific antibodies, originally developed by Roche, has also been utilized for HIV-1 antibody development in recent years. The CrossMAb format allows for correct assembly of two heavy chains and two light chains from different antibodies into one bispecific antibody molecule that resembles a typical monoclonal antibody in terms of mass and architecture, and with no artificial linkers required (Fig. 1d) [46]. This is achieved by combining the knob-into-hole technology, which enables heterodimerization of two different heavy chains, and the light chain crossover technology, which ensures correct association of each of the light chains with their cognate heavy chains.

In one study, CrossMAb antibodies targeting four major HIV-1 Env epitopes known to be important for
HIV-1 neutralization, the CD4 binding site, V3 glycan, V1V2, and MPER regions, were engineered [47]. These HIV-1 CrossMAb bispecific antibodies neutralized 95-97\% of circulating HIV-1 strains tested, and the most promising candidate from this study, VRC07-PG9-16, neutralized the panel of viruses with a median $\mathrm{IC}_{50}$ of $0.055 \mu \mathrm{g} / \mathrm{mL}$. This represented an improvement in neutralization breadth and coverage over the single parental mAbs from which VRC07-PG9-16 was derived, and was similar in breadth and potency to the co-administration of the two parental mAbs, which was not the case for all of the bispecific antibodies engineered and evaluated.

\section{Engineering trispecific antibodies with improved breadth}

It is now well known that the highly dynamic nature of HIV-1 replication in vivo demands treating HIV-1 with three antiretroviral agents simultaneously since viral escape against any single agent is an inevitable consequence of the large number of HIV-1 mutants generated per day within an infected person [48-50]. With this in mind, the continuous evolution of HIV-1 Env during the course of infection also attests to the exceptional selective pressure exerted by naturally elicited virus-specific antibodies [51]. Therefore, trispecific antibodies with the potential to inhibit viral entry with three distinct HIV-1 
Env-targeting antibody moieties was of interest. In one study, the trispecific antibodies VRC01/PGDM140010E8v4 and N6/PGDM1400-10E8v4 were engineered using a knob-in hole $(\mathrm{KiH})$ heterodimerization technology [52] and a cross-over dual variable immunoglobulin G (CODV-Ig) technology to ensure affinity of each variable region was maintained [53] in order to target the HIV-1 envelope CD4 binding site, MPER and V2 glycan site (Fig. 1e) [54]. Multiple combinations of broadly neutralizing parental antibodies and formats were tested before downselecting VRC01/PGDM1400-10E8v4 and N6/PGDM1400-10E8v4, which demonstrated 98\% and $>99 \%$ breadth, respectively, as defined by $\geq 50 \%$ neutralization. Surface plasmon resonance confirmed that each of the three antibody-targeting domains within VRC01/ PGDM1400-10E8v4 had comparable affinities for its HIV-1 Env antigens relative to its parental Fab counterparts. VRC01/PGDM1400-10E8v4 also provided 100\% protection to nonhuman primates challenged mucosally with a mixture of two SHIVs, SHIV 325C and SHIV BaLP4, which each had varying sensitivities to two of the parental mAb counterparts of VRC01/PGDM1400$10 \mathrm{E} 8 \mathrm{v} 4$, while only $62 \%$ and $75 \%$ of nonhuman primates administered VRC01 or PGDM1400, respectively, were protected in this model. Therefore, the improvement in neutralization breadth observed by VRC01/PGDM1400$10 \mathrm{E} 8 \mathrm{v} 4$ in vitro translated to an improved breadth of protection against SHIV in vivo.

While the bispecific and trispecific antibodies discussed above enhanced HIV-1 neutralization breadth relative to their parental $\mathrm{mAb}$ counterparts, they were limited in their ability to enhance potency relative to the parental mAbs provided individually or in combination. This is thought to be due, in part, to the low spike density of gp160 trimers on the surface of HIV-1 [55-57], which may limit the ability of these bispecific and trispecific antibodies to bind to the HIV-1 envelope bivalently (or trivalently in the case of a trispecific antibody) through inter-spike crosslinking. The gp160 trimer spike structure itself may also limit the ability of these multi-specific antibodies to achieve intra-spike crosslinking $[55,56]$. While antibodies elicited naturally during HIV-1 infection also typically interact monovalently with the HIV-1 gp160 trimer spike, polyreactive antibodies have been proposed to be positively selected and retained during affinity maturation and can increase their overall apparent affinity for HIV-1 Env through heteroligation [58]. The VRC07-PG9-16 CrossMAb discussed earlier can achieve a potency similar to, but not better than, the most potent of its parental mAbs against any particular virus, and this is thought to be due to an inability of VRC07-PG9-16 to simultaneously bind both of its epitopes on the HIV-1 Env trimer [47]. If multivalent binding of these bispecific or trispecific antibodies was possible, one could imagine that a significant enhancement in antiviral potency could be gained in addition to enhanced breadth.

\section{Engineering multi-specific antibodies to improve breadth and potency against HIV-1}

One study has investigated the importance of this potential for enhanced HIV-1 neutralization by inter- and intra-spike binding by using DNA as a "molecular ruler" that has a HIV-1 Env binding antibody domain conjugated to each end [59]. By increasing or decreasing the number of basepairs (bp) between two Fabs of either 3BNC60 [6] or VRC01 [10], homo-dimer Fabs with different lengths of "reach" were used to probe the distance needed to achieve avidity as opposed to single arm Fab binding. These studies revealed that a length of $\sim 60$ bp resulted in $\sim 100$-fold increased potency for either 3BNC60 or VRC01 homo-diFabs against the specific HIV-1 strain tested, likely due to bivalent binding to two CD4 binding sites within a single gp120 trimer. Hetero-diFabs also exhibited enhanced potency as compared to combinations of their monoclonal antibody counterparts. For example, a PG16-3BNC60 diFab, targeting both V1V2 and the CD4 binding site in a single gp120 trimer, enhanced neutralization potency by $\sim 100$ fold when a 50 bp double-stranded (ds) DNA bridge was used to separate these two Fabs. The 50-60 bp ds DNA bridges in these molecules represent a reach distance of $\sim 17-21 \mathrm{~nm}$ between the two Fabs in a single molecule, which is longer than the $\sim 12-15 \mathrm{~nm}$ reach of two Fab arms in a typical IgG molecule [55]. While the molecular flexibility and dynamics that may be associated with an antibody binding to either the open or closed HIV-1 Envelope trimer may somewhat alter these distances in a case-dependent manner, it is generally thought that the reach between the two Fab arms in a HIV-1 multi-specific antibody would need to be larger than that within a typical IgG in order to capture the benefits of avidity and multivalent binding. These DNA diFab constructs provide an elegant method to investigate the science underlying antibody avidity to HIV-1 Env, but are not readily translatable to product development and clinical use.

All of the bispecific antibodies discussed until now have utilized an IgG1 or IgG4 subtype, based on their intended mechanism of action. Another subclass, IgG3, possesses a relatively longer and more flexible hinge domain region $[60,61]$, which may allow for the greater "reach" needed to achieve bivalent binding of a bispecific antibody against HIV-1 Env. To test this, a small panel of CrossMAb format HIV-1 bispecific antibodies were generated in which the typical IgG1 hinge domain was replaced with a longer and more flexible IgG3 hingelike region called IgG3C- (Fig. 1f) [62]. One of these 
IgG3C- hinge variants that targeted the CD4 binding site and V3 region of the HIV-1 envelope, 3BNC117/ PGT135, exhibited both superior breadth (93\% as defined by $50 \%$ inhibition and $89.1 \%$ as defined by $80 \%$ inhibition) and superior potency $\left(\mathrm{IC}_{50}\right.$ geometric mean of $0.036 \mu \mathrm{g} /$ $\mathrm{mL}$ and $\mathrm{IC}_{80}$ geometric mean of $0.159 \mu \mathrm{g} / \mathrm{mL}$ ) relative to its single parental $\mathrm{mAbs}$ or the predicted combination of both parental mAbs. Variants in which the IgG3C- hinge length of 3BNC117/PGT135 were decreased resulted in decreased neutralization activity. Combined with structural data modeling 3BNC117 and PGT135 Fabs complexed with the Env trimer, this suggests that the IgG3C- hinge variant of 3BNC117/PGT135 may allow for bivalent binding, enhanced avidity, and ultimately greater potency relative to its parental mAb counterparts. No differences in the pharmacokinetic profile of this bispecific antibody were observed in mice in comparison to typical mAbs, and a $1.5 \log _{10}$ decrease in viral load was observed in a humanized mouse model for HIV-1 treatment. In comparison, treatment with a mixture of the 3BNC117 and PGT135 parental mAbs yielded very little change in viral loads.

Another study reported the engineering of trispecific antibodies in order to increase "reach" and improve HIV-1 neutralization breadth and potency. Using scFv domains connected in tandem with flexible linkers, different formats of scFv domains targeting the HIV-1 CD4 binding site, V3, and MPER regions were engineered and characterized for their ability to improve antiviral activity and HIV-1 Env binding avidity (Fig. 1g). From these studies, 10E8v4/PGT121-VRC01 emerged as the most promising trispecific antibody candidate, exhibiting 99.5\% breadth, as defined by $50 \%$ inhibition, an $\mathrm{IC}_{50}$ geometric mean of 0.069 , and an $\mathrm{IC}_{80}$ geometric mean of $0.298 \mu \mathrm{g} /$ $\mathrm{mL}$ [63]. Biolayer interferometry was used to confirm that all three scFv domains in this trispecific antibody could bind to their cognate HIV-1 Env epitopes, and it is suggested that the four-fold enhancement in potency of 10E8v4/PGT121-VRC01 relative to its parental mAbs is due to the cooperative effect of binding to at least two epitopes simultaneously on the HIV-1 Env trimer.

In addition to bispecific and trispecific antibody formats, smaller Fc fusion proteins have also been engineered with the goal of improving potency by enabling bispecific avidity. $4 \mathrm{Dm} 2 \mathrm{~m}$ is comprised of a single domain of soluble CD4, known as $\mathrm{mD} 1.22$, fused to the $\mathrm{N}$ - and C-termini of a human IgG1 heavy chain constant region, and an antibody domain targeting the coreceptor binding site on gp120, known as m36.4, fused to the $\mathrm{N}$-terminus of the human antibody light chain constant region via a glycine-serine linker (Fig. 1h) [64, 65]. This bispecific multivalent fusion protein neutralized all HIV-1 isolates tested with a potency about 10-fold higher than the CD4 binding site antibody, VRC01. The authors reasoned that the improvement in potency between $4 \mathrm{Dm} 2 \mathrm{~m}$ and a variant with $\mathrm{m} 36.4$ only at the N-termini, known as $2 \mathrm{Dm} 2 \mathrm{~m}$, was due to bivalent binding of both the head and tail m36.4 antibody domains in 4Dm2 $\mathrm{m}$ and the relative close proximity of the CD4 binding site and coreceptor binding site on gp120.

eCD4-Ig is a fusion of CD4-Ig, which itself is comprised of CD4 domains 1 and 2 fused to Fc, and a small CCR5mimetic sulfopeptide (Fig. 1i) [66]. eCD4-Ig neutralized $100 \%$ of a diverse panel of circulating HIV-1 strains, and could also neutralize HIV-2 strains, and this outstanding antiviral breadth is thought to be due to the relatively well conserved nature of the CD4 binding site and CCR5 coreceptor binding site epitopes on HIV-1 Env. A structural model of eCD4-Ig bound to the HIV-1 Env trimer predicts that both the CD4-Ig and CCR5-mimetic sulfopeptide bind avidly and cooperatively to HIV-1. This would support the high potency of eCD4-Ig, neutralizing a panel of HIV-1 with a geometric mean of $<0.05 \mu \mathrm{g} / \mathrm{mL}$, as defined by $50 \%$ inhibition. eCD4-Ig variants neutralized each particular HIV-1 strain tested with a potency 10 - to $>200$-fold better than CD4-Ig alone. A rhesus version of one of the bispecific fusion variants, known as rh-eCD4-IgG2 $2^{\mathrm{I39N}, \mathrm{mim} 2}$, was cloned into an adenoassociated virus serotype 2 (AAV2) vector and, when coadministered with a separate single-stranded AAV vector expressing rhesus tyrosine-protein sulfotransferase to promote rh-eCD4-Ig sulfation, provided $100 \%$ protection against repeated SHIV-AD8 challenges. Recently, an improved variant of eCD4-Ig that utilized $\mathrm{mD} 1.22$, the stabilized form of CD4 domain 1 discussed earlier, was shown to improve the potency of this bispecific fusion peptide by another 9 -fold while maintaining satisfactory production efficiency [67].

The antibodies discussed above demonstrate the principle that engineering multi-specific antibodies against HIV-1 for increased avidity can increase their antiviral potency and breadth. However, the large divergence in HIV-1 Envs and their relative dynamic nature pose a challenge to identifying multi-specific molecules with sufficient reach to consistently interact with target epitopes across diverse HIV-1 strains. Another approach to increase avidity and potency is to exploit the dynamic nature of HIV-1 Env to identify at least two antiviral targets in the overall viral entry process. By investigating the spatiotemporal process of HIV-1 entry, it was plausible that new combinations of bispecific antibody targets could be discovered that were not exclusive to targeting HIV-1 Env.

PG9-iMab and PG16-iMab, comprised of the scFv of the V1V2-targeting PG9 or PG16 mAbs fused to the CD4-targeting mAb ibalizumab, are two such examples 
(Fig. 1b) [68]. PG9-iMab and PG16-iMab both exhibited impressive breadth and potency, neutralizing $100 \%$ of viruses tested, as defined by $50 \%$ inhibition. When defined as $80 \%$ inhibition, PG9-iMab still neutralized $100 \%$ of viruses while PG16-iMab neutralized $98 \%$ of viruses. The enhancement in potency was also remarkable, with PG9-iMab exhibiting an $\mathrm{IC}_{50}$ geometric mean of $0.004 \mu \mathrm{g} / \mathrm{mL}$ and an $\mathrm{IC}_{80}$ geometric mean of $0.017 \mu \mathrm{g} /$ $\mathrm{mL}$, and PG16-iMab exhibiting an $\mathrm{IC}_{50}$ geometric mean of $0.003 \mu \mathrm{g} / \mathrm{mL}$ and an $\mathrm{IC}_{80}$ geometric mean of $0.015 \mu \mathrm{g} /$ $\mathrm{mL}$. The enhancement in potency was $>20$-fold compared to the parental mAb ibalizumab and $>100$-fold compared to the parental mAb PG9 or PG16, and far better than a co-mixture of the two parental mAbs together. Importantly, the ability of PG9-iMab to bind both CD4 on the T cell and V1V2 on HIV-1 Env did not result in any obviously detrimental form of crosslinking that could enhance viral activity in the TZM-bl and PBMC neutralization assays evaluated, but rather only potently and broadly inhibited viral activity. In some cases, the potencies of these bispecific antibodies were improved up to four-logs compared to their parental mAb counterparts. Mechanistic studies determined that the enhanced potency of PG9-iMab required anchoring of this bispecific antibody to $\mathrm{CD} 4$ via its ibalizumab component. Additional modeling studies suggest that this anchoring to CD4 positions the PG9 scFv component of PG9-iMab so that it can more easily interact with the V1V2 epitope on the Env of the incoming viral particle. In effect, this increases the local concentration of PG9 $\mathrm{scFv}$ precisely at the site where it can exert its antiviral activity.

Interestingly, the enhancement in potency observed with PG9-iMab in this $\mathrm{scFv}$ bispecific format was not replicated with other $\mathrm{scFv}$ bispecific combinations such as VRC01-iMab, 3BNC60-iMab or 45-46-iMab, which target CD4 via ibalizumab and the HIV-1 Env CD4 binding site via VRC01, 3BNC60, or NIH45-46 scFv domains [44]. However, an enhancement in potency was observed with the CD4- and HIV-1 Env V3-targeting PGT123iMab, PGT128-iMab and 10-1074-iMab, approaching the level of potency observed with PG9-iMab or PG16iMab. This suggests that, similar to a preferred accessibility to the HIV-1 Env V1V2 epitope when PG9-iMab and PG16-iMab are anchored to CD4, the HIV-1 Env V3 epitope may be similarly accessible when PGT123-iMab, PGT128-iMab or 10-1074-iMab are bound to CD4 [44].

While several scFv-format bispecific antibodies are currently in development, several properties inherent to this bispecific antibody format must be addressed before they can be advanced into the clinic. For example, the linker fusing the $\mathrm{V}_{\mathrm{H}}$ and $\mathrm{V}_{\mathrm{L}}$ domains of the scFv moiety, and the linker fusing the scFv moiety to either an IgG-like molecule or another scFv moiety, must be sufficiently flexible so as not to impair the normal folding and function of the binding domains within the bispecific antibody, must be sufficiently stable so as to avoid cleavage and subsequent separation of the antibody binding domains during manufacture or in vivo, and must be sufficiently soluble so as to avoid potential aggregation. The ideal linker length and orientation of the $V_{H}$ and $V_{L}$ domains within the scFv moiety may also vary depending on the biophysical properties and mechanism of action of the particular bispecific antibody. All of these properties vary from molecule to molecule, and must be empirically investigated and optimized during the development process. Finally, the unnatural architecture of many scFv-format bispecific antibodies, which may deviate significantly from typical IgG antibodies, or their associated linkers, may create neoantigens or expose cryptic epitopes that may lead to immunogenicity in vivo [69]. While several in silico or in vitro methods may be able to identify potential hotspots of antibody immunogenicity, host immune responses cannot be predicted solely by these methods [70], and the ultimate test of antibody immunogenicity is by clinical study [71].

As discussed earlier, the CrossMAb bispecific antibody format retains more of a native IgG-like structure and avoids the need for foreign linker sequences [46], which may obviate some of the development challenges associated with scFv bispecific antibodies. However, the nativelike structure of CrossMAbs may also restrict the "reach," and consequently the avidity, of two HIV-1 Env epitope binding variable domains when incorporated into this format [47]. Directing bispecific antibodies to host cell receptors with one of the CrossMAb arms, however, while targeting the other CrossMAb arm to the HIV-1 envelope, could take advantage of the dynamic nature of the HIV-1 entry process and allow for avidity by binding two HIV-1 entry targets simultaneously, similar to what was achieved with the PG9-iMab scFv format bispecific antibody. One study constructed and characterized a panel of 20 CrossMAb bispecific antibodies in which one arm inhibited HIV-1 by targeting the CD4 receptor or the CCR5 coreceptor via ibalizumab (iMab) or PRO140 (P140) [23, 24], and the other arm targeted the HIV-1 envelope MPER, CD4 binding site, V3 region, V1V2 region, or gp41-gp120 interface via 10E8, 3BNC117, PGT128, PGT145 or PGT151 [1, 3, 6, 8], and an optimal combination was identified which yielded exquisite antiviral potency and breadth [25]. The HIV-1 CrossMAbs 10E8/iMab and 10E8/P140 exhibited $\mathrm{IC}_{50}$ geometric means of $0.002 \mu \mathrm{g} / \mathrm{mL}$ and $0.001 \mu \mathrm{g} / \mathrm{mL}$, respectively, and neutralization breadth (as assessed by $\geq 50 \%$ neutralization) of $100 \%$ and $99 \%$, respectively. This represented a synergistic enhancement in potency hundreds of fold greater than those of its parental mAbs, and represented 
some of the most potent bispecific antibodies against HIV-1 identified to date. Interestingly, a CrossMAb comprised of a CD4-targeting ibalizumab arm and a V1V2targeting PGT145 arm did not enhance antiviral potency, even though the CD4/V1V2-targeting PG9-iMab yielded a synergistic enhancement in potency in a scFv bispecific format [68]. Based on structural modeling data of the PG9-iMab scFv bispecific antibody discussed earlier, it is possible that the PG9 moiety may not be positioned at the right angle or length to neutralize HIV-1 Env when it is bound to CD4 or CCR5 in a CrossMAb format. Both 10E8/iMab and 10E8/P140 CrossMAbs, similar to the PG9-iMab scFv bispecific antibody, exerted their impressive antiviral activity by anchoring 10E8 near the two receptors HIV-1 utilizes, CD4 and CCR5, essentially placing 10E8 at precisely the right place and right time to bind HIV-1 Env MPER and potently neutralize an incoming viral particle. Indeed, if either the 10E8 or ibalizumab arm in 10E8/iMab (or the 10E8 or PRO140 arm in 10E8/ $\mathrm{P} 140$ ) was engineered for reduced binding, the antiviral activity of the mutant bispecific was only as good as the $\mathrm{mAb}$ represented by the remaining intact arm within each of the bispecific CrossMAbs. After several rounds of antibody engineering to identify variants of these HIV-1 CrossMAbs with improved physicochemical homogeneity, an optimized variant known as $10 \mathrm{E} 8_{\mathrm{V} 2.0} / \mathrm{iMab}$ (renamed 10E8.2/iMab) emerged with improved physicochemical properties, two-fold enhancement in bioavailability, and further improvement in antiviral potency compared to its predecessor $\left(\mathrm{IC}_{50}\right.$ geometric mean of $0.002 \mu \mathrm{g} / \mathrm{mL}$ and $\mathrm{IC}_{80}$ geometric mean of $0.006 \mu \mathrm{g} / \mathrm{mL}$ ). 10E8.2/iMab also demonstrated impressive antiviral activity in vivo, reducing viral load in HIV-1-infected humanized mice by $1.7 \log _{10}$ and providing $100 \%$ protection against multiple systemic challenges with the tier-2 R5 virus, JR-CSF. Utilizing in vitro neutralization data for 10E8.2/iMab and other HIV-1 mAbs against subtype $\mathrm{A}, \mathrm{C}$, and $\mathrm{D}$ pseudoviruses, a model of neutralization potency and breadth for single and two mAb combinations predicted that this single bispecific molecule, 10E8.2/iMab, could provide broader and more potent protection across subtypes as compared to all two $\mathrm{mAb}$ combinations evaluated [22].

\section{Bispecific antibody development challenges}

The impressive potency, breadth and higher barrier against emerging resistant viruses that can be achieved with HIV-1 bispecific or trispecific antibodies warrants their further investigation. In addition, the ability to capture this impressive antiviral activity in a single multi-specific molecule, as opposed to combinations of multiple mAbs, makes the development of HIV-1 bispecific and trispecific antibodies an attractive path commercially. One HIV-1 multi-specific molecule could achieve the same or better antiviral activity as combinations of multiple mAbs, but the manufacturing, storage, transport and administration costs remain similar to that of a single agent.

However, while the manufacturing process for typical mAbs is relatively mature and established, unexpected manufacturing challenges unique to each bispecific or trispecific antibody format must be overcome in order to make development of these multi-specific molecules a feasible strategy for HIV-1 treatment or prevention. Some of the challenges of scFv format bispecific antibodies were discussed earlier, such as the potential for linker instability, aggregation propensity and potential immunogenicity in vivo due to the difference in architecture between these bispecific molecules and typical IgG antibodies. Additionally, the non-native structure of this bispecific antibody format could result in a poor pharmacokinetic profile in vivo. Other bispecific formats, such as the CrossMAb format, avoid the use of linkers and maintain a more natural IgG antibody architecture while still achieving bispecificity as asymmetric IgG heterodimers. However, because two distinct heavy chains and two distinct light chains are required to produce the desired product, homodimer byproducts or light chain mispairings may arise and must be overcome.

Downstream processes may also possess unique challenges. While typical mAbs are purified using a Protein $A$ resin that binds to the $\mathrm{Fc}$ region of the $\mathrm{mAb}$, and then additional purification polishing steps are performed as necessary, bispecific antibodies that utilize asymmetry, such as the CrossMAb format, cannot be distinguished from homodimer impurities since the Fc regions of both the target heterodimer product and the impurity consisting of homodimers would interact equally well with Protein A. These bispecific formats must exploit asymmetry to their advantage in their purification processes as well, for example, by using a kappa light chain with one arm of the intact molecule and a lambda light chain with the other arm of the intact molecule so that successive rounds of purification that capture each of the light chain arms sequentially would allow for purification of the intact molecule [42]. Other purification tools that can take advantage of asymmetry could also be employed, such as engineering each bispecific antibody arm with sufficient differences in isolectric points so that sequential purification by anion exchange and cation exchange chromatographies would result in purified heterodimers. Additionally, the combination of difficult upstream production procedures for certain complex bispecific antibody formats and multiple downstream purification steps 
may result in lower final product yields for bispecific antibodies as compared to typical mAbs.

Nonetheless, the tremendous therapeutic potential of HIV-1 bispecific and trispecific antibodies, with evidence of synergistically enhancing antiviral activity by several logs and the potential for drastically lower production costs by containing the therapeutic to a singular molecular entity, necessitate strategies be developed to overcome these challenges. By embarking on a scientifically rigorous approach towards developability and manufacturability that combines elements of quality by design with a deep mechanistic understanding of the specific therapeutic, promising bispecific or trispecific antibodies can overcome these developmental hurdles in order to advance into human testing as novel and potentially powerful therapeutic or prophylactic agents against HIV1. Indeed, several of these novel candidates are already in clinical development (Figs. 1 and 5). Below, we present a case study of one such bispecific antibody against HIV-1.

\section{Case study: quality by design approach to engineer a HIV-1 bispecific antibody with improved developability properties}

As discussed earlier, 10E8.2/iMab [25] is a CrossMAb format bispecific antibody in which one antigen binding arm (iMab) targets the human CD4 receptor via the Fab of the humanized mAb ibalizumab [23], and a second antigen binding arm (10E8.2) targets the HIV-1 Env MPER via a variant of the human mAb 10E8 (Fig. 1d) [3]. The positioning of CD4- and MPER-targeting arms in this CrossMAb format produces a bispecific antibody with exquisitely potent and broad HIV-1 antiviral activity, neutralizing $100 \%$ of circulating HIV-1 strains in a 118 multi-clade panel with an $\mathrm{IC}_{50}$ geometric mean of $0.002 \mu \mathrm{g} / \mathrm{mL},>97 \%$ of this panel with an $\mathrm{IC}_{80}$ geometric mean of $0.006 \mu \mathrm{g} / \mathrm{mL}$, and $>98 \%$ of a second 200 virus Clade $C$ panel with similar antiviral potencies [25]. 10E8.2/iMab also potently inhibited HIV-1 in vivo, reducing viral load in HIV-1-infected humanized mice by $1.7 \log _{10}$ and providing $100 \%$ protection against systemic challenge with a tier-2 R5 virus [25].

Despite this impressive antiviral activity in vitro and in vivo, a short-term "stress test" of 10E8.2/iMab revealed that this bispecific antibody starts to precipitate soon after incubation at $50{ }^{\circ} \mathrm{C}$, suggesting a potential thermoinstability and aggregation propensity of this molecule under certain conditions. Five different CrossMAb format bispecific antibodies are currently in the clinic [72-76], indicating that the CrossMAb technology itself is not the cause of this thermoinstability and aggregation propensity. Additionally, other iMab-based CrossMAbs and the ibalizumab mAb did not exhibit such a high level of thermoinstability, indicating that this arm of 10E8.2/
iMab was likely not causing this issue. However, the parental mAb 10E8 was previously reported to have poor solubility and a tendency to precipitate [77], suggesting that the MPER-binding arm in 10E8.2/iMab was most likely responsible for the insolubility observed at high temperatures. This inherent biophysical property had the potential to limit the further development of this potent bispecific antibody.

Hydrophobic residues constantly or dynamically exposed on the surface of proteins often result in aggregation as protein concentration increases [31, 78]. Therefore, a quality by design (QbD) approach was taken to identify and systematically mutate externally-facing hydrophobic residues on the 10E8.2 arm of 10E8.2/iMab and to replace them with hydrophilic residues in an effort to find a functional variant with improved solubility. Out of 17 antibody variants engineered, hydrophobic to hydrophilic mutations at 6 residues in 10E8.2/iMab retained satisfactory functional activity, and combinations of these 6 mutations were subjected to biophysical characterizations to determine if there was any improvement in solubility.

The apparent solubility of 10E8.4/iMab was determined in comparison to $10 \mathrm{E} 8.2$ /iMab by formulating both antibodies at identical starting concentrations and subjecting them to ultracentrifugation. At concentrations above $50 \mathrm{mg} / \mathrm{mL}, 10 \mathrm{E} 8.4 /$ iMab showed consistently higher protein concentrations and solubility over time as compared to $10 \mathrm{E} 8.2 / \mathrm{iMab}$, and the apparent solubility, or maximum concentration achieved, of 10E8.4/iMab was calculated to be $>230 \mathrm{mg} / \mathrm{mL}$ (Fig. 2a). This improvement in solubility, combined with long-term stability data, strongly suggests that 10E8.4/iMab could be formulated not just for intravenous administration to humans, but also at the higher concentrations required for subcutaneous administration since volume constraints are often a concern for delivery by this latter route. Consequently, 10E8.4/iMab delivery by both of these routes of administration will be evaluated clinically.

The turbidity of 10E8.2/iMab and 10E8.4/iMab at various protein concentrations was also evaluated in order to draw a correlation between these two parameters. While the turbidity of both 10E8.2/iMab and 10E8.4/ iMab expectedly increased with protein concentration over time, 10E8.2/iMab showed consistently higher turbidity than $10 \mathrm{E} 8.4 / \mathrm{iMab}$ at the same protein concentrations over $100 \mathrm{mg} / \mathrm{mL}$, indicating improved solubility of 10E8.4/iMab (Fig. 2b). 10E8.2/iMab and 10E8.4/iMab were also subjected to a forced degradation analysis to determine their relative protein stabilities under thermal stress-inducing conditions. In addition to an improvement in appearance and decrease in turbidity, 10E8.4/ iMab also exhibited better intact molecule purity over 

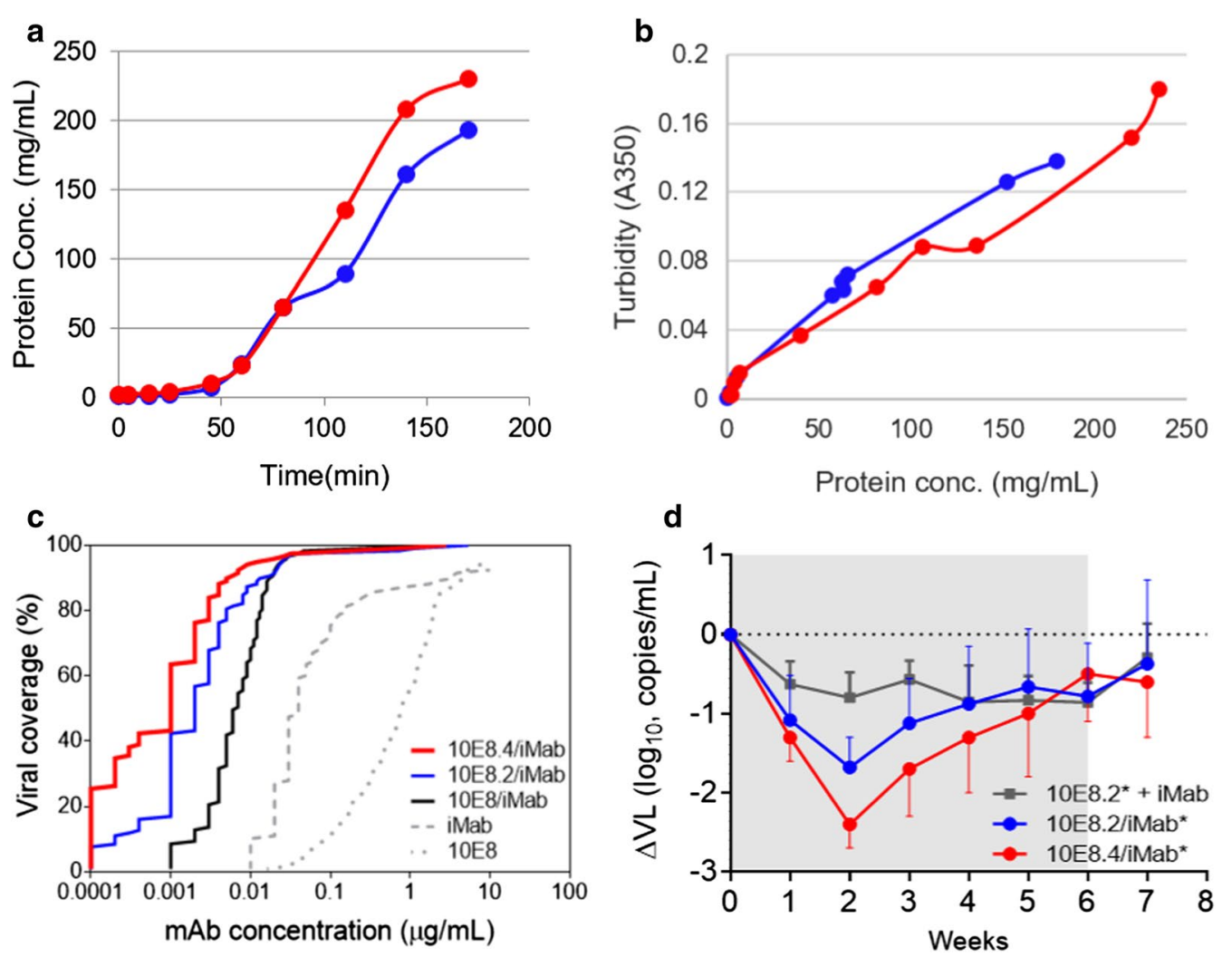

Fig. 2 Improved solubility and antiviral activity of 10E8.4/iMab. a Apparent solubility and $\mathbf{b}$ turbidity of 10E8.4/iMab and its predecessor variant 10E8.2/iMab. c Percent of a 118 Tier-2 HIV-1 Env pseudovirus panel neutralized (based on $1 C_{50}$ values) by 10E8.4/iMab and its predecessor variants 10E8/iMab and 10E8.2/iMab. Parental mAbs iMab and 10E8 are included for reference. $\mathbf{d}$ Decrease in viral load by 10E8.4/iMab and its predecessor variant, 10E8.2/iMab, in HIV-1-infected humanized mice. Shaded area indicates the period of weekly antibody administration. Error bars $=$ SD. * = N297A mutant variant of each bispecific antibody. As reported previously [84], this mutation in the Fc region of each bispecific antibody is required for evaluation of non-FcR binding human antibodies in the murine model

time by capillary electrophoresis (CE) SDS-PAGE and fewer aggregation-associated high molecular weight species over time by size exclusion chromatography, indicating its relatively better stability under thermal stress-inducing conditions as compared to 10E8.2/iMab.

In addition to its improved solubility and thermostability, 10E8.4/iMab also exhibited a 2.5-fold enhancement in antiviral potency when tested against the same panel of 118 Tier-2 HIV-1 pseudotyped viruses representing diverse clades and geographic origins described earlier (Fig. 2c). In a humanized mouse model of HIV-1 infection, weekly administrations of 10E8.4/iMab reduced the viral load of HIV-1-infected mice by $2.4 \log _{10}$ while a maximum mean viral load reduction of $\sim 1.7 \log _{10}$ was observed in mice treated with 10E8.2/iMab (Fig. 2d).

In summary, in silico analysis of the 10E8.2/iMab sequence and structure for potential aggregation-inducing hotspots revealed a number of residues that could be detrimental for the developability of this potent bispecific antibody for the clinic. A potential setback as a result of these inherent molecular properties may often not be realized until significant funds and time are exerted for the advancement of a particular therapeutic into the clinic. However, utilizing a $\mathrm{QbD}$ approach to systematically mutate each of these hotspot residues individually, and iteratively testing combinations of these engineered variants for improved product quality attributes, led to the identification of a new improved variant, 10E8.4/iMab. While there is always the theoretical risk that engineering new residues into an antibody may result in unanticipated immunogenicity, the likelihood of this is uncertain and cannot be definitively assessed until clinical investigation [71]. Therefore, based on its superior solubility and stability and its further improved potent in vitro and vivo antiviral activity, 10E8.4/ iMab was selected as a clinical lead candidate for further development. 


\section{Case study: cell line development of a CrossMAb format HIV-1 bispecific antibody}

Cell line development in preparation for reproducible production of a given $\mathrm{mAb}$ therapeutic for human use is now an established process, as evidenced by the $>85$ mAbs approved for commercial use by the US FDA for the treatment of a number of different human diseases [79], and this does not include the many more mAbs that are currently in preclinical and clinical development. The heavy chain and light chain of a given mAb are encoded together on one plasmid that contains an antibiotic resistance selection marker or separately on two plasmids, each with its own unique antibiotic resistance selection marker. These plasmids are then stably transfected into a cell line. After transfection, single clones that produce high titers of the $\mathrm{mAb}$, as determined by Protein A binding to the Fc region of antibody secreted into the supernatant, are selected and further characterized in order to downselect a lead clone for GMP master cell bank production. For the cell line development of 10E8.4/iMab, a modified approach was necessary due to a total of four separate open reading frames (encoding 10E8.4 heavy chain, 10E8.4 light chain, iMab heavy chain, and iMab light chain) that need to be stably transfected. By transient transfection, encoding four different open reading frames in four separate plasmids reproducibly produces CrossMAb bispecific antibodies with $>80 \%$ intact molecule purity [46]. For stable transfection, however, encoding these four different open reading frames in four separate plasmids was not feasible because the high level of antibiotic selection pressure against four distinct markers would drastically reduce the number of surviving clones that could be screened for high titerproducing antibody levels.

After attempting stable transfection of 10E8.4/iMab encoded in two or three plasmid configurations, and screening for high titer clones by Protein A binding to the Fc region of the secreted antibody, the highest level of intact molecule purity produced from a stable pool of clones was $68.5 \%$, which is too low to support a viable upstream production and downstream purification strategy for clinical development. Analysis by non-reduced CE SDS-PAGE of the impurities present in the supernatant of the top stable pools revealed a significant fraction of heavy chain-heavy chain $(\mathrm{HH})$ and heavy chainheavy chain-light chain (HHL) impurities present in the clonal supernatant. Theoretically, the knob-in-hole and light chain crossover technologies incorporated into the CrossMAb format should prevent these impurities from being secreted. However, our investigational analyses revealed that, if all four ORFs are not present in the transfection mix, impure byproducts can be readily secreted. For example, transfection of 10E8.4 HC and iMab HC, without their cognate light chains, can be secreted (Fig. 3a), as can 10E8.4 HC, iMab HC and iMab LC impurities (Fig. 3b). Fundamental biological studies of monoclonal antibody secretion indicate that antibody HCs are not typically secreted from cells without their cognate LCs associated, and a closer investigation revealed that the signal for this antibody secretion is associated with close proximity of the $\mathrm{CH} 1$ domain of a nascently formed antibody $\mathrm{HC}$ with the CL domain of a nascently formed antibody LC in the endoplasmic reticulum [80]. Due to the unique configuration of the light chain crossover technology in CrossMAb antibodies, however, the CL

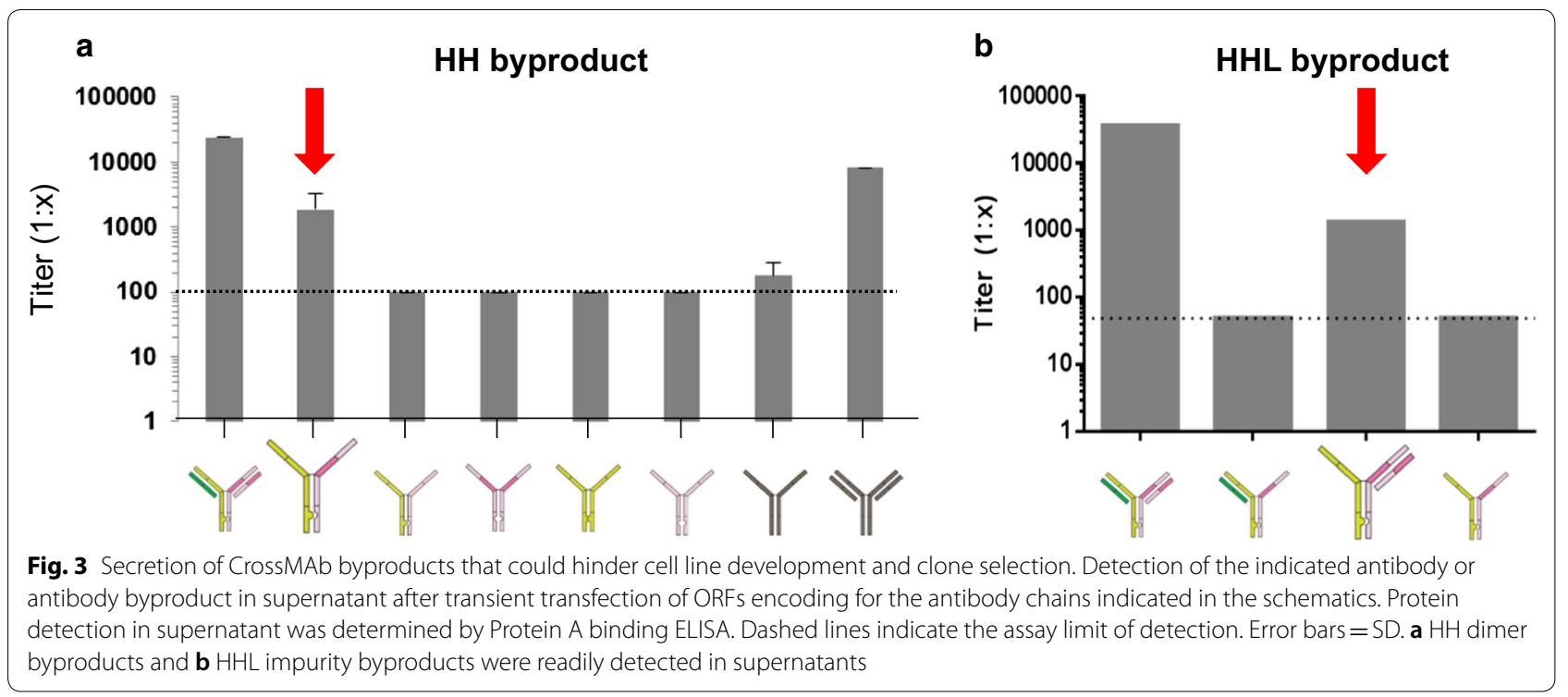


a

Intact Molecule

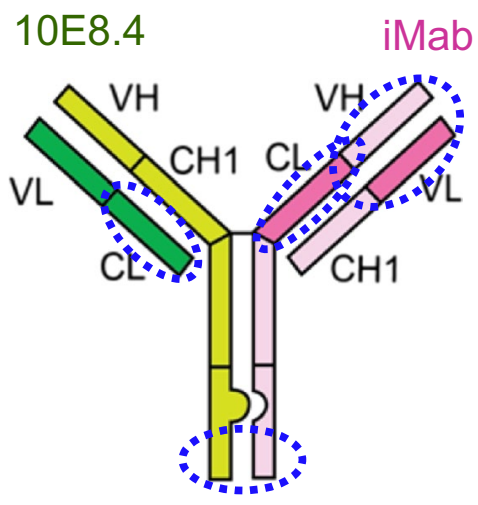

b

\section{Byproducts}

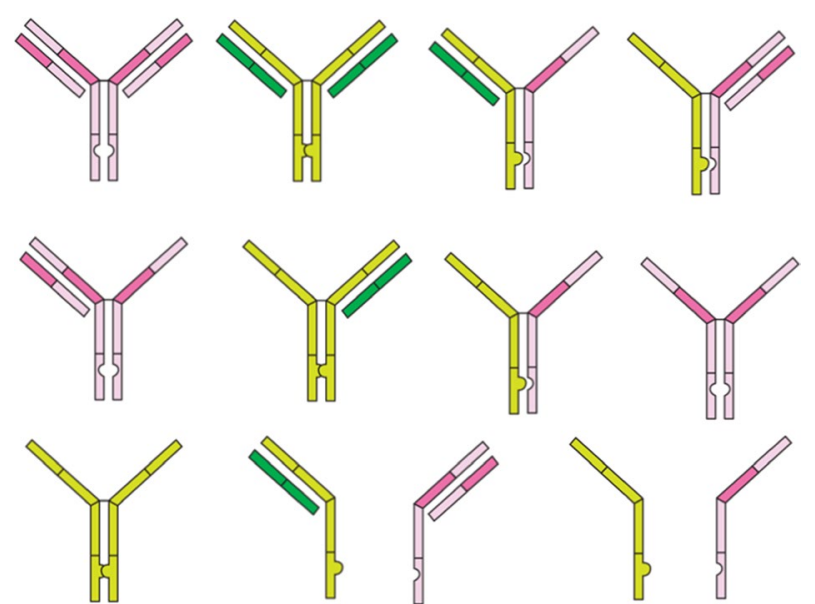

Fig. 4 CrossMab format for bispecific antibody production. Knob-in hole mutations in the $\mathrm{CH} 3$ domains favor heterodimer heavy chain formation. $\mathrm{CH} 1-\mathrm{CL}$ crossover in one arm of the CrossMAb favors proper light chain association with its cognate heavy chain. In combination, a intact molecule production and secretion is favored and $\mathbf{b}$ byproduct production and secretion is disfavored. Dashed blue circles indicate target domains that, when detected simultaneously, ensure a greater percentage of intact molecule

of ibalizumab is located on the "heavy chain" (Fig. 4a), and we speculate that the close proximity of this CL in the ibalizumab "heavy chain" and the $\mathrm{CH} 1$ domain in the 10E8.4 HC can trigger antibody secretion without their cognate LCs. With consideration to our stable cell line transfection efforts, one can easily envision how overexpression or underexpression of one or more of the four bispecific antibody chains in a stable cell line could allow for permissive secretion of $\mathrm{HH}$ or HHL impurities if the missing chain(s) is produced at relatively low levels. Also, since our initial screening strategy, which is commonly used for $\mathrm{mAb}$ cell line selection, indiscriminately selected for high-producer clones by Fc-binding to Protein A, it was impossible to differentiate clones producing the intact HHLL molecule from those that produced HHLL along with a mixture of $\mathrm{HH}$ and $\mathrm{HHL}$ impurities since all of these products would have nearly identical binding properties to Protein A.

To overcome these challenges, we undertook a multipronged approach that specifically addressed the unique differences in cell line development between a typical $\mathrm{mAb}$ and the 10E8.4/iMab bispecific CrossMAb. We generated new two and three plasmid vector combinations encoding the 10E8.4 HC, 10E8.4 LC, iMab $\mathrm{HC}$ and iMab LC in several different permutations, and transiently transfected them at numerous ratios to identify the plasmid combinations and ratios that could give the best percentage of intact molecule purity by transient transfection in order to downselect the most promising set of plasmids and conditions to advance into stable transfection studies. In all, more than 20 different plasmid configurations and conditions were evaluated. Next, by designing a new screening strategy that recognized four distinct domains of $10 \mathrm{E} 8.4 / \mathrm{iMab}$ simultaneously rather than only its singular Fc region, we could select for high titer producing clones with better assurance that they were producing fully intact HHLL molecules rather than byproduct impurities (Fig. 4a). In effect, if we equate identifying a high titer producing clone within a large pool of stably transfected clones to identifying a needle in a haystack, our redesigned screening strategy was a powerfully tuned magnet that could sift through the "hay" of clones to find our high titer producing "needle." To do this, we developed new FRET-based methods to simultaneously detect multiple distinct arms within the 10E8.4/ iMab intact molecule, and utilized CE SDS-PAGE as our analytical screening tool to confirm intact molecule purity levels relative to byproduct impurities. If a suitable bispecific ELISA-based method was available that could simultaneously detect both functional antibody arms, this could also be employed. Finally, we plated and screened over five times as many clones as was done for a typical mAb cell line development program in order to ensure that we could identify a suitable lead clone. In effect, now equipped with our powerful screening strategy and magnet, we could increase the size of the haystack in order to ensure that one or more of our needles was contained within it. These laborious efforts proved fruitful, and a final lead cell line clone 
was identified that produced 10E8.4/iMab at $>90 \%$ intact molecule purity after a simple 1 -step purification and at a titer of $>3 \mathrm{~g} / \mathrm{L}$. This titer is on par with excellent $\mathrm{mAb}$-producing clones and much better than what is expected for a typical bispecific antibody. Additional polishing steps purified 10E8.4/iMab to $>97 \%$, which is well within the range of purity acceptable to advance this novel and potent HIV-1 bispecific antibody into clinical evaluation.

\section{Conclusions}

The new generation of broadly neutralizing mAbs against HIV-1 has given the field a new avenue of hope for prophylactic and therapeutic possibilities to reduce the existing HIV-1 burden. In addition to the recent FDA approval of ibalizumab (Trogarzo ${ }^{\circledR}$ ) for use as salvage therapy in patients whose viruses are resistant to multiple existing antiretroviral drugs, VRC01 is currently in two Phase $2 \mathrm{~b}$ efficacy trials for HIV-1 prevention in HIV-1 uninfected men and transgender persons who have sex with men in the United States, Peru, Brazil, and Switzerland (HVTN 704/HPTN 085) and in HIV-1 uninfected sexually active women in seven countries in sub-Saharan Africa (HVTN 703/HPTN 081) [26, 81]. Known as the Antibody Mediated Prevention
(AMP) Studies, the lessons learned from these VRC01 Phase $2 b$ efficacy trials will be of tremendous benefit the field of antibody-mediated HIV-1 prevention. It is clear, however, that drastic improvements to antibody potency and breadth will be required in order to produce a feasible antibody regimen which could be used widespread and which could limit the emergence of viral resistance well known to those in the HIV-1 treatment field. Bispecific and trispecific antibodies offer a new beacon of hope to combat viral resistance by improving neutralization breadth and, in some cases, by drastically improving antiviral potency by orders of magnitude over the best HIV-1 mAbs currently in existence (Fig. 5). However, the development of these HIV-1 multi-specific antibodies is not without its own challenges. The potential for aggregation, immunogenicity and low GMP cell line titers is an issue for any antibody, and these are amplified in cases of multi-specific antibodies due to their unique formats and engineered properties required to create their multi-specificity. In addition to the challenges discussed in this review, other downstream chemistry, manufacturing and controls obstacles such as antibody purification and stability of engineered multi-specific molecules may exist. Further in development, nonclinical challenges, such

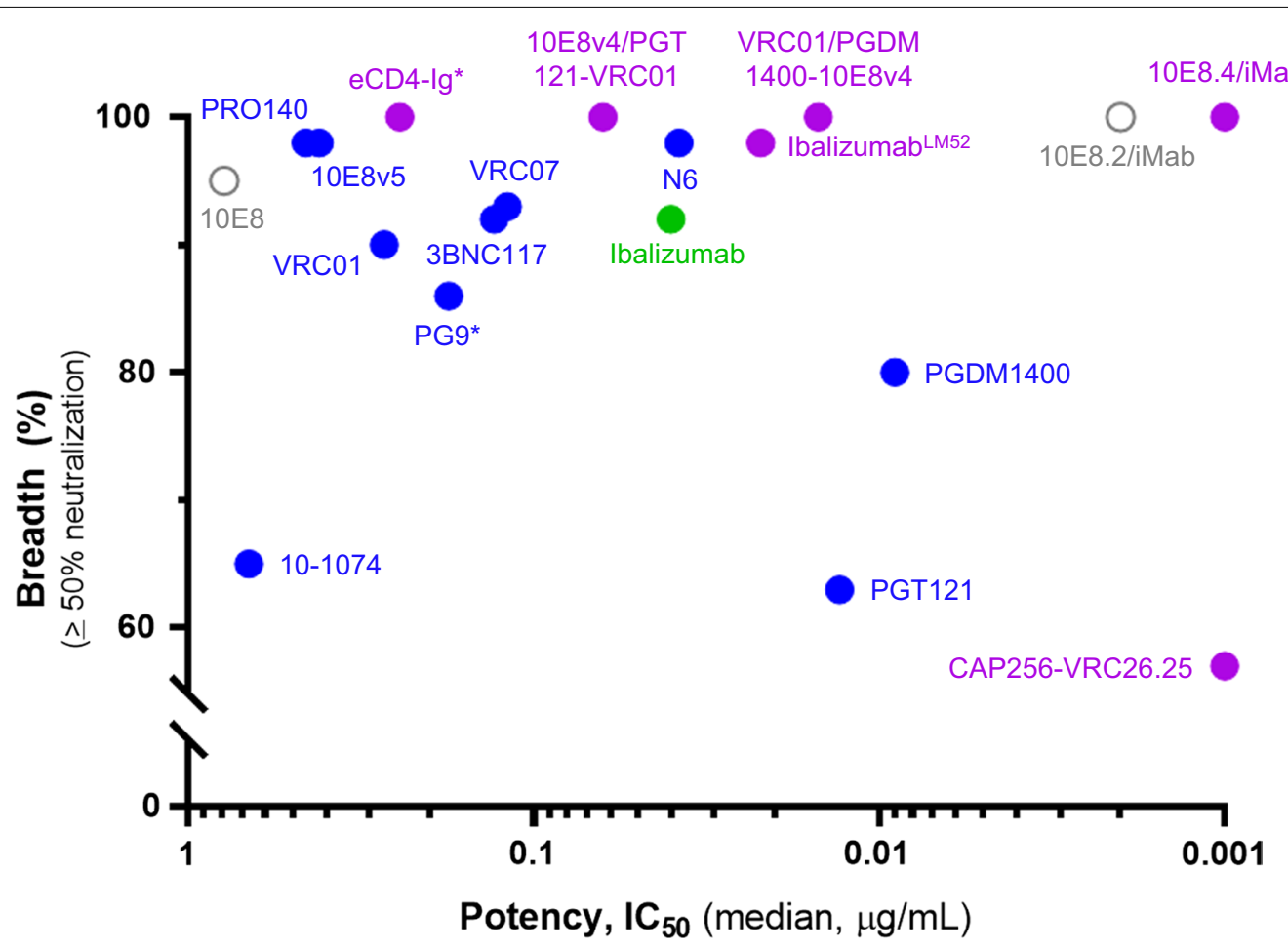

Fig. 5 Antiviral potency and breadth of HIV-1 mAbs and multi-specific Abs. HIV-1 mAbs and multi-specific antibodies that are licensed (green), in clinical trials (blue), or in clinical development (purple). Open circles represent earlier variants of antibodies in development that are presented. *Means antibodies were delivered by AAV. Figure adapted from Xu et al., 2017 and additional published reports [54, 85, 86] 
as manufacturing and incorporating parental $\mathrm{mAb}$ control groups into GLP toxicology programs in the event that safety signals for a given multi-specific antibody requires further investigation, may also arise [82]. During clinical investigation, pharmacokinetic and anti-drug antibody assays must be able to detect each specificity within a given multi-specific antibody, and therefore reagents or assays that can detect each unique epitope within a given HIV-1 multi-specific antibody are preferred [83].

Despite these challenges, the tremendous opportunities for bispecific and trispecific antibodies against HIV-1 are readily evident. Applying the same creativity and rigor to the development and manufacture of HIV-1 multi-specific antibodies as that which was used for their creation and initial characterization promises to offer to the field a new generation of potent and broad multi-specific antibodies that could be ready to enter the clinic within the same timeframe as a typical $\mathrm{mAb}$. In parallel, the ongoing discovery of ever more potent and broadly neutralizing HIV-1 mAbs continues to provide new and improved foundational starting blocks for incorporation into multi-specific antibodies. How we create and advance these powerful multispecific antibodies for the prevention and treatment of HIV-1 will only be limited by our imagination, rigor and diligence.

\footnotetext{
Abbreviations

AAV: adeno-associated virus; bp: basepairs; CE: capillary electrophoresis; CODV-Ig: cross-over dual variable immunoglobulin G; DNA: deoxyribonucleic acid; ds: double-stranded; Env: envelope; HH: heavy chain-heavy chain; HHL: heavy chain-heavy chain-light chain; IC: inhibitory concentration; HIV-1: human immunodeficiency virus 1; KiH: knob-in hole; iMab: ibalizumab; mAb: monoclonal antibody; MPER: membrane proximal external region; P140: PRO140; QbD: quality by design; SHIV: simian human immunodeficiency virus; scFv: single-chain variable fragment.
}

\section{Authors' contributions}

NNP, JY, YH and DDH jointly wrote this review article and conducted or supervised all unpublished studies presented. All authors read and approved the final manuscript.

\section{Acknowledgements \\ The authors wish to thank Wuxi Biologics for their support in 10E8.2/iMab and 10E8.4/iMab solubility and thermostability studies and 10E8.4/iMab cell line development studies, Christian Klein at Roche Innovation Center Zurich for helpful discussions regarding 10E8.4/iMab cell line development and for information regarding clinical experience with CrossMAbs, Michael Seaman at Beth Israel Deaconess Medical Center, Harvard Medical School for conduct- ing in vitro neutralization studies of 10E8.4/iMab against the multi-clade HIV envelope pseudotyped virus panel, Mili Gajjar for technical assistance with 10E8.4/iMab in vivo studies, Yang Luo for project coordination, Wendy Chen for help with figure preparation, and members of the Ho laboratory for helpful discussions.}

\section{Competing interests}

$J Y, Y H$ and DDH are inventors on a patent describing HIV CrossMAbs, including 10E8/iMab and 10E8/P140 and their variants, for HIV-1 prevention and therapy.
The rights of this technology for use in HIV-1 therapy have been licensed to TaiMed Biologics, Inc., which also owns the commercial rights to ibalizumab. DDH is the scientific founder of TaiMed Biologics, Inc. and, in this capacity, has equity in the company. DDH is also an inventor on a patent describing LM52, which has been licensed to TaiMed Biologics, Inc.

\section{Availability of data and materials}

The data presented in this review were obtained primarily from previous publications as indicated in the references cited along with the respective data. Any newly analyzed datasets presented in this review will be made available from the corresponding author upon reasonable request.

\section{Consent to participate}

Not applicable.

\section{Consent for publication}

Not applicable.

\section{Ethics approval}

Animals used to generate previously unpublished data were bred and maintained at the Comparative Bioscience Center of The Rockefeller University in accordance with the regulations of its Institutional Animal Care and Use Committee (IACUC). Studies were conducted under protocols approved by this committee and in compliance with the Animal Welfare Act and other federal statutes and regulations relating to animals and experiments involving animals.

\section{Funding}

DDH is supported by the Bill and Melinda Gates Foundation's Collaboration for AIDS Vaccine Discovery for the development of bispecific antibodies for HIV-1 prevention under Grants OPP1040731 and OPP1169162.

\section{Publisher's Note}

Springer Nature remains neutral with regard to jurisdictional claims in published maps and institutional affiliations.

Received: 13 June 2018 Accepted: 11 August 2018

Published online: 29 August 2018

\section{References}

1. Blattner C, Lee JH, Sliepen K, Derking R, Falkowska E, de la Pena AT, Cupo A, Julien JP, van Gils M, Lee PS, et al. Structural delineation of a quaternary, cleavage-dependent epitope at the gp41-gp120 interface on intact HIV-1 Env trimers. Immunity. 2014;40:669-80.

2. Doria-Rose NA, Schramm CA, Gorman J, Moore PL, Bhiman JN, DeKosky BJ, Ernandes MJ, Georgiev IS, Kim HJ, Pancera M, et al. Developmental pathway for potent V1V2-directed HIV-neutralizing antibodies. Nature. 2014;509:55-62.

3. Huang J, Ofek G, Laub L, Louder MK, Doria-Rose NA, Longo NS, Imamichi H, Bailer RT, Chakrabarti B, Sharma SK, et al. Broad and potent neutralization of HIV-1 by a gp41-specific human antibody. Nature. 2012;491:406-12.

4. Mouquet $\mathrm{H}$, Scharf L, Euler Z, Liu Y, Eden C, Scheid JF, Halper-Stromberg A, Gnanapragasam PN, Spencer DI, Seaman MS, et al. Complex-type $\mathrm{N}$-glycan recognition by potent broadly neutralizing HIV antibodies. Proc Natl Acad Sci USA. 2012;109:E3268-77.

5. Rudicell RS, Kwon YD, Ko SY, Pegu A, Louder MK, Georgiev IS, Wu X, Zhu J, Boyington JC, Chen $X$, et al. Enhanced potency of a broadly neutralizing HIV-1 antibody in vitro improves protection against lentiviral infection in vivo. J Virol. 2014;88:12669-82.

6. Scheid JF, Mouquet H, Ueberheide B, Diskin R, Klein F, Oliveira TY, Pietzsch J, Fenyo D, Abadir A, Velinzon K, et al. Sequence and structural convergence of broad and potent HIV antibodies that mimic CD4 binding. Science. 2011;333:1633-7.

7. Sok D, van Gils MJ, Pauthner M, Julien JP, Saye-Francisco KL, Hsueh J, Briney B, Lee JH, Le KM, Lee PS, et al. Recombinant HIV envelope trimer selects for quaternary-dependent antibodies targeting the trimer apex. Proc Natl Acad Sci USA. 2014;111:17624-9. 
8. Walker LM, Huber M, Doores KJ, Falkowska E, Pejchal R, Julien JP, Wang SK, Ramos A, Chan-Hui PY, Moyle M, et al. Broad neutralization coverage of HIV by multiple highly potent antibodies. Nature. 2011;477:466-70.

9. Walker LM, Phogat SK, Chan-Hui PY, Wagner D, Phung P, Goss JL, Wrin T, Simek MD, Fling S, Mitcham JL, et al. Broad and potent neutralizing antibodies from an African donor reveal a new HIV-1 vaccine target. Science. 2009:326:285-9.

10. Wu X, Yang ZY, Li Y, Hogerkorp CM, Schief WR, Seaman MS, Zhou T, Schmidt SD, Wu L, Xu L, et al. Rational design of envelope identifies broadly neutralizing human monoclonal antibodies to HIV-1. Science. 2010;329:856-61.

11. Caskey M, Klein F, Lorenzi JC, Seaman MS, West AP Jr, Buckley N, Kremer $G$, Nogueira L, Braunschweig M, Scheid JF, et al. Viraemia suppressed in HIV-1-infected humans by broadly neutralizing antibody 3BNC117. Nature. 2015;522:487-91.

12. Caskey M, Schoofs T, Gruell H, Settler A, Karagounis T, Kreider EF, Murrell B, Pfeifer N, Nogueira L, Oliveira TY, et al. Antibody 10-1074 suppresses viremia in HIV-1-infected individuals. Nat Med. 2017;23:185-91.

13. Ledgerwood JE, Coates EE, Yamshchikov G, Saunders JG, Holman L, Enama ME, DeZure A, Lynch RM, Gordon I, Plummer S, et al. Safety, pharmacokinetics and neutralization of the broadly neutralizing HIV-1 human monoclonal antibody VRCO1 in healthy adults. Clin Exp Immunol. 2015;182:289-301.

14. Lynch RM, Boritz E, Coates EE, DeZure A, Madden P, Costner P, Enama ME, Plummer S, Holman L, Hendel CS, et al. Virologic effects of broadly neutralizing antibody VRCO1 administration during chronic HIV-1 infection. Sci Transl Med. 2015;7:319ra206.

15. A Clinical Trial of PGDM1400 and PGT121 Monoclonal Antibodies in HIVinfected and HIV-uninfected Adults. ClinicalTrials.gov. U.S. National Library of Medicine. https://clinicaltrials.gov/ct2/show/NCT03205917. Accessed 13 May 2018

16. VRC 605: Safety and Pharmacokinetics of a Human Monoclonal Antibody, VRC-HIVMAB075-00-AB (VRC07-523IS), Administered Intravenously or Subcutaneously to Healthy Adults. ClinicalTrials.gov. U.S. National Library of Medicine. https://clinicaltrials.gov/ct2/show/NCT03015181. Accessed 13 May 2018.

17. A Clinical Trial of the Safety, Pharmacokinetics and Antiviral Activity of PGT121 Monoclonal Antibody (mAb) in HIV-uninfected and HIV-infected Adults. ClinicalTrials.gov. U.S. National Library of Medicine. https://clini caltrials.gov/ct2/show/NCT02960581. Accessed 13 May 2018.

18. Jacobson JM, Saag MS, Thompson MA, Fischl MA, Liporace R, Reichman RC, Redfield RR, Fichtenbaum CJ, Zingman BS, Patel MC, et al. Antiviral activity of single-dose PRO 140, a CCR5 monoclonal antibody, in HIVinfected adults. J Infect Dis. 2008;198:1345-52.

19. Toma J, Weinheimer SP, Stawiski E, Whitcomb JM, Lewis ST, Petropoulos CJ, Huang W. Loss of asparagine-linked glycosylation sites in variable region 5 of human immunodeficiency virus type 1 envelope is associated with resistance to CD4 antibody ibalizumab. J Virol. 2011;85:3872-80.

20. Pace CS, Fordyce MW, Franco D, Kao CY, Seaman MS, Ho DD. Anti-CD4 monoclonal antibody ibalizumab exhibits breadth and potency against HIV-1, with natural resistance mediated by the loss of a V5 glycan in envelope. J Acquir Immune Defic Syndr. 2013;62:1-9.

21. Wagh K, Bhattacharya T, Williamson C, Robles A, Bayne M, Garrity J, Rist M, Rademeyer C, Yoon H, Lapedes A, et al. Optimal combinations of broadly neutralizing antibodies for prevention and treatment of HIV-1 clade C infection. PLoS Pathog. 2016;12:e1005520.

22. Wagh $K$, Seaman MS, Zingg M, Fitzsimons T, Barouch DH, Burton DR, Connors M, Ho DD, Mascola JR, Nussenzweig MC, et al. Potential of conventional \& bispecific broadly neutralizing antibodies for prevention of HIV-1 subtype A, C \& D infections. PLoS Pathog. 2018;14:e1006860.

23. Burkly LC, Olson D, Shapiro R, Winkler G, Rosa JJ, Thomas DW, Williams C, Chisholm P. Inhibition of HIV infection by a novel CD4 domain 2-specific monoclonal antibody. Dissecting the basis for its inhibitory effect on HIVinduced cell fusion. J Immunol. 1992;149:1779-87.

24. Trkola A, Ketas TJ, Nagashima KA, Zhao L, Cilliers T, Morris L, Moore JP, Maddon PJ, Olson WC. Potent, broad-spectrum inhibition of human immunodeficiency virus type 1 by the CCR5 monoclonal antibody PRO 140. JVirol. 2001;75:579-88.

25. Huang Y, Yu J, Lanzi A, Yao X, Andrews CD, Tsai L, Gajjar MR, Sun M, Seaman MS, Padte NN, Ho DD. Engineered bispecific antibodies with exquisite HIV-1-neutralizing activity. Cell. 2016;165:1621-31.
26. Emu B, Fessel J Schrader S, Kumar P, Richmond G, Win S, Weinheimer S, Marsolais C, Lewis S. Phase 3 study of ibalizumab for multidrug-resistant HIV-1. N Engl J Med. 2018:379:645-54.

27. Markham A. Ibalizumab: first global approval. Drugs. 2018;78:781-5.

28. A Randomized, Double-blind, Placebo-controlled Trial, Followed by Single-arm Treatment of PRO 140 in Combination w/Optimized Background Therapy in Treatment-Experienced HIV Subjects (PRO 140). ClinicalTrials. gov. U.S. National Library of Medicine. https://clinicaltrials.gov/ct2/show/ NCT02483078?term=PRO140\&rank=2. Accessed 11 May 2018.

29. Morris L, Mkhize NN. Prospects for passive immunity to prevent HIV infection. PLoS Med. 2017;14:e1002436.

30. Diskin R, Scheid JF, Marcovecchio PM, West AP Jr, Klein F, Gao H, Gnanapragasam PN, Abadir A, Seaman MS, Nussenzweig MC, Bjorkman PJ. Increasing the potency and breadth of an HIV antibody by using structure-based rational design. Science. 2011;334:1289-93.

31. Kwon YD, Georgiev IS, Ofek G, Zhang B, Asokan M, Bailer RT, Bao A, Caruso W, Chen X, Choe M, et al. Optimization of the solubility of HIV-1-neutralizing antibody $10 \mathrm{E} 8$ through somatic variation and structure-based design. J Virol. 2016;90:5899-914.

32. VRC 610: Phase I Safety and Pharmacokinetics Study to Evaluate a Human Monoclonal Antibody (MAB) VRC-HIVMAB095-00-AB (10E8VLS) Administered Alone or Concurrently with MAB VRC- HIVMAB075-00-AB (VRC07-523LS) via Subcutaneous Injection in Healthy Adults. ClinicalTrials.gov. U.S. National Library of Medicine. https://clinicaltrials.gov/ct2/ show/NCT03565315. Accessed 03 Aug 2018.

33. Kwon YD, Chuang GY, Zhang B, Bailer RT, Doria-Rose NA, Gindin TS, Lin B, Louder MK, McKee K, O'Dell S, et al. Surface-matrix screening identifies semi-specific interactions that improve potency of a near pan-reactive HIV-1-neutralizing antibody. Cell Rep. 2018;22:1798-809.

34. Freeman MM, Seaman MS, Rits-Volloch S, Hong X, Kao CY, Ho DD, Chen B. Crystal structure of HIV-1 primary receptor CD4 in complex with a potent antiviral antibody. Structure. 2010;18:1632-41.

35. Song $R$, Franco D, Kao CY, Yu F, Huang Y, Ho DD. Epitope mapping of ibalizumab, a humanized anti-CD4 monoclonal antibody with anti-HIV-1 activity in infected patients. J Virol. 2010;84:6935-42.

36. Song R, Oren DA, Franco D, Seaman MS, Ho DD. Strategic addition of an $\mathrm{N}$-linked glycan to a monoclonal antibody improves its HIV-1-neutralizing activity. Nat Biotechnol. 2013;31:1047-52.

37. Product Pipeline:TMB-360/365. TaiMed Biologics. http://www.taimedbiol ogics.com/pipeline/36. Accessed 18 May 2018.

38. Karpovsky B, Titus JA, Stephany DA, Segal DM. Production of targetspecific effector cells using hetero-cross-linked aggregates containing anti-target cell and anti-Fc gamma receptor antibodies. J Exp Med. 1984;160:1686-701.

39. Perez P, Hoffman RW, Shaw S, Bluestone JA, Segal DM. Specific targeting of cytotoxic T cells by anti-T3 linked to anti-target cell antibody. Nature. 1985:316:354-6.

40. Staerz UD, Kanagawa O, Bevan MJ. Hybrid antibodies can target sites for attack by T cells. Nature. 1985;314:628-31.

41. Chames P, Baty D. Bispecific antibodies for cancer therapy: the light at the end of the tunnel? MAbs. 2009;1:539-47.

42. Brinkmann U, Kontermann RE. The making of bispecific antibodies. MAbs. 2017.9.182-212.

43. Sun M, Pace CS, Yao X, Yu F, Padte NN, Huang Y, Seaman MS, Li Q, Ho DD. Rational design and characterization of the novel, broad and potent bispecific HIV-1 neutralizing antibody iMabm36. J Acquir Immune Defic Syndr. 2014;66:473-83.

44. Song R, Pace C, Seaman MS, Fang $Q$, Sun M, Andrews CD, Wu A, Padte NN Ho DD. Distinct HIV-1 neutralization potency profiles of ibalizumab-based bispecific antibodies. J Acquir Immune Defic Syndr. 2016;73:365-73.

45. Zhou T, Georgiev I, Wu X, Yang ZY, Dai K, Finzi A, Kwon YD, Scheid JF, Shi W, Xu L, et al. Structural basis for broad and potent neutralization of HIV-1 by antibody VRC01. Science. 2010;329:811-7.

46. Schaefer W, Regula JT, Bahner M, Schanzer J, Croasdale R, Durr H, Gassner C, Georges G, Kettenberger H, Imhof-Jung S, et al. Immunoglobulin domain crossover as a generic approach for the production of bispecific IgG antibodies. Proc Natl Acad Sci USA. 2011;108:11187-92.

47. Asokan M, Rudicell RS, Louder M, McKee K, O'Dell S, Stewart-Jones G, Wang K, Xu L, Chen X, Choe M, et al. Bispecific antibodies targeting different epitopes on the HIV-1 envelope exhibit broad and potent neutralization. J Virol. 2015;89:12501-12. 
48. Ho DD, Neumann AU, Perelson AS, Chen W, Leonard JM, Markowitz M Rapid turnover of plasma virions and CD4 lymphocytes in HIV-1 infection. Nature. 1995:373:123-6.

49. Perelson AS, Neumann AU, Markowitz M, Leonard JM, Ho DD. HIV-1 dynamics in vivo: virion clearance rate, infected cell life-span, and viral generation time. Science. 1996;271:1582-6.

50. Wei X, Ghosh SK, Taylor ME, Johnson VA, Emini EA, Deutsch P, Lifson JD, Bonhoeffer S, Nowak MA, Hahn BH, et al. Viral dynamics in human immunodeficiency virus type 1 infection. Nature. 1995:373:117-22.

51. Haynes BF, Shaw GM, Korber B, Kelsoe G, Sodroski J, Hahn BH, Borrow P, McMichael AJ. HIV-host interactions: implications for vaccine design. Cell Host Microbe. 2016;19:292-303.

52. Merchant AM, Zhu Z, Yuan JQ, Goddard A, Adams CW, Presta LG, Carter P. An efficient route to human bispecific lgG. Nat Biotechnol. 1998;16:677-81

53. Steinmetz A, Vallee F, Beil C, Lange C, Baurin N, Beninga J, Capdevila C, Corvey C, Dupuy A, Ferrari P, et al. CODV-lg, a universal bispecific tetravalent and multifunctional immunoglobulin format for medical applications. MAbs. 2016:8:867-78.

54. Xu L, Pegu A, Rao E, Doria-Rose N, Beninga J, McKee K, Lord DM, Wei RR, Deng G, Louder M, et al. Trispecific broadly neutralizing HIV antibodies mediate potent SHIV protection in macaques. Science. 2017:358:85-90.

55. Klein JS, Gnanapragasam PN, Galimidi RP, Foglesong CP, West AP Jr, Bjorkman PJ. Examination of the contributions of size and avidity to the neutralization mechanisms of the anti-HIV antibodies b12 and 4E10. Proc Natl Acad Sci USA. 2009;106:7385-90.

56. Liu J, Bartesaghi A, Borgnia MJ, Sapiro G, Subramaniam S. Molecular architecture of native HIV-1 gp120 trimers. Nature. 2008;455:109-13.

57. Zhu P, Liu J, Bess J Jr, Chertova E, Lifson JD, Grise H, Ofek GA, Taylor KA Roux KH. Distribution and three-dimensional structure of AIDS virus envelope spikes. Nature. 2006:441:847-52.

58. Mouquet H, Scheid JF, Zoller MJ, Krogsgaard M, Ott RG, Shukair S, Artyomov MN, Pietzsch J, Connors M, Pereyra F, et al. Polyreactivity increases the apparent affinity of anti-HIV antibodies by heteroligation. Nature. 2010:467:591-5.

59. Galimidi RP, Klein JS, Politzer MS, Bai S, Seaman MS, Nussenzweig MC, West AP Jr, Bjorkman PJ. Intra-spike crosslinking overcomes antibody evasion by HIV-1. Cell. 2015;160:433-46.

60. Roux KH, Strelets L, Brekke OH, Sandlie I, Michaelsen TE. Comparisons of the ability of human $\lg G 3$ hinge mutants, $\lg M$, $\lg E$, and $\lg \mathrm{A} 2$, to form small immune complexes: a role for flexibility and geometry. J Immunol. 1998:161:4083-90.

61. Roux KH, Strelets L, Michaelsen TE. Flexibility of human IgG subclasses. J Immunol. 1997;159:3372-82.

62. Bournazos S, Gazumyan A, Seaman MS, Nussenzweig MC, Ravetch JV. Bispecific anti-HIV-1 antibodies with enhanced breadth and potency. Cell. 2016;165:1609-20.

63. Steinhardt JJ, Guenaga J, Turner HL, McKee K, Louder MK, O’Dell S, Chiang $\mathrm{Cl}$, Lei L, Galkin A, Andrianov AK, et al. Rational design of a trispecific antibody targeting the HIV-1 Env with elevated anti-viral activity. Nat Commun. 2018:9:877.

64. Chen W, Bardhi A, Feng Y, Wang Y, Qi Q, Li W, Zhu Z, Dyba MA, Ying T, Jiang $\mathrm{S}$, et al. Improving the $\mathrm{CH} 1-\mathrm{CK}$ heterodimerization and pharmacokinetics of 4Dm2 m, a novel potent CD4-antibody fusion protein against HIV-1. MAbs. 2016:8:761-74.

65. Chen W, Feng Y, Prabakaran P, Ying T, Wang Y, Sun J, Macedo CD, Zhu Z, HeY, Polonis VR, Dimitrov DS. Exceptionally potent and broadly crossreactive, bispecific multivalent HIV-1 inhibitors based on single human CD4 and antibody domains. J Virol. 2014;88:1125-39.

66. Gardner MR, Kattenhorn LM, Kondur HR, von Schaewen M, Dorfman T, Chiang JJ, Haworth KG, Decker JM, Alpert MD, Bailey CC, et al. AAVexpressed eCD4-Ig provides durable protection from multiple SHIV challenges. Nature. 2015;519:87-91.

67. Fetzer I, Gardner MR, Davis-Gardner ME, Prasad NR, Alfant B, Weber JA Farzan M. eCD4-Ig variants that more potently neutralize HIV-1. J Virol. 2018:92:e02011-17.

68. Pace CS, Song R, Ochsenbauer C, Andrews CD, Franco D, Yu J, Oren DA, Seaman MS, Ho DD. Bispecific antibodies directed to CD4 domain 2 and HIV envelope exhibit exceptional breadth and picomolar potency against HIV-1. Proc Natl Acad Sci USA. 2013;110:13540-5.
69. Miller LL, Korn EL, Stevens DS, Janik JE, Gause BL, Kopp WC, Holmlund JT, Curti BD, Sznol M, Smith JW 2nd, et al. Abrogation of the hematological and biological activities of the interleukin-3/granulocyte-macrophage colony-stimulating factor fusion protein PIXY321 by neutralizing antiPIXY321 antibodies in cancer patients receiving high-dose carboplatin. Blood. 1999;93:3250-8.

70. Gokemeijer J, Jawa V, Mitra-Kaushik S. How close are we to profiling immunogenicity risk using in silico algorithms and in vitro methods? An industry perspective. AAPS J. 2017:19:1587-92.

71. Guidance for Industry: Immunogenicity Assessment for Therapeutic Protein Products. U.S. Department of Health and Human Services. Food and Drug Administration. Center for Drug Evaluation and Research (CDER). Center for Biologics Evaluation and Research (CBER). 2014.

72. Bacac M, Fauti T, Sam J, Colombetti S, Weinzierl T, Ouaret D, Bodmer W, Lehmann S, Hofer T, Hosse RJ, et al. A novel carcinoembryonic antigen T-cell bispecific antibody (CEA TCB) for the treatment of solid tumors. Clin Cancer Res. 2016;22:3286-97.

73. Brunker P, Wartha K, Friess T, Grau-Richards S, Waldhauer I, Koller CF, Weiser B, Majety M, Runza V, Niu H, et al. RG7386, a novel tetravalent FAP-DR5 antibody, effectively triggers FAP-dependent, avidity-driven DR5 hyperclustering and tumor cell apoptosis. Mol Cancer Ther. 2016;15:946-57.

74. Chakravarthy U, Bailey C, Brown D, Campochiaro P, Chittum M, Csaky K, Tufail A, Yates P, Cech P, Giraudon M, Delmar P, Szczesny P, Sahni J, Boulay A, Nagel S, Fürst-Recktenwald S, Schwab D. Phase I trial of anti-vascular endothelial growth factor/anti-angiopoietin 2 bispecific antibody RG7716 for neovascular age-related macular degeneration. Ophthalmol Retina. 2017;1:474-85.

75. Regula JT, Lundh von Leithner P, Foxton R, Barathi VA, Cheung CM, Bo Tun SB, Wey YS, Iwata D, Dostalek M, Moelleken J, et al. Targeting key angiogenic pathways with a bispecific CrossMAb optimized for neovascular eye diseases. EMBO Mol Med. 2016;8:1265-88.

76. Tabernero J, Melero I, Ros W, Argiles G, Marabelle A, Rodriguez-Ruiz ME, Albanell J, Calvo E, Moreno V, Cleary JM, Eder JP, Karanikas V, Bouseida S, Sandoval F, Sabanes D, Sreckovic S, Hurwitz H, Paz-Ares LG, Saro Suarez $J M$, Segal NH. Phase la and lb studies of the novel carcinoembryonic antigen (CEA) T-cell bispecific (CEA CD3 TCB) antibody as a single agent and in combination with atezolizumab: preliminary efficacy and safety in patients with metastatic colorectal cancer (mCRC). J Clin Oncol. 2017;35:3002.

77. Anti-HIV-1 gp41 Monoclonal (10E8), Catalog Number: 12294. NIH AIDS Reagent Program. https://www.aidsreagent.org/reagentdetail. $\mathrm{cfm} ? \mathrm{t}=$ monoclonal_antibodies\&id=680. Accessed 27 May 2018.

78. Chennamsetty N, Voynov V, Kayser V, Helk B, Trout BL. Design of therapeutic proteins with enhanced stability. Proc Natl Acad Sci USA. 2009;106:11937-42

79. Drugs@FDA: FDA approved drug products. U.S. Food and Drug Administration. https://www.accessdata.fda.gov/scripts/cder/daf. Accessed 18 May 2018.

80. Feige MJ, Groscurth S, Marcinowski M, Shimizu Y, Kessler H, Hendershot LM, Buchner J. An unfolded $\mathrm{CH} 1$ domain controls the assembly and secretion of IgG antibodies. Mol Cell. 2009;34:569-79.

81. Gilbert PB, Juraska M, deCamp AC, Karuna S, Edupuganti S, Mgodi N, Donnell DJ, Bentley C, Sista N, Andrew P, et al. Basis and statistical design of the passive HIV-1 antibody mediated prevention (AMP) test-of-concept efficacy trials. Stat Commun Infect Dis. 2017;9:20160001.

82. Points to Consider in the Manufacture and Testing of Monoclonal Antibody Products for Human Use. U.S. Department of Health and Human Services. Food and Drug Administration. Center for Biologics Evaluation and Research (CBER). 1997.

83. Guidance for Industry: Assay Development and Validation for Immunogenicity Testing of Therapeutic Protein Products. U.S. Department of Health and Human Services. Food and Drug Administration. Center for Drug Evaluation and Research (CDER). Center for Biologics Evaluation and Research (CBER). Center for Devices and Radiological Health (CDRH). 2016.

84. Chao DT, Ma X, Li O, Park H, Law D. Functional characterization of N297A, a murine surrogate for low-Fc binding anti-human CD3 antibodies. Immunol Invest. 2009;38:76-92.

85. Doria-Rose NA, Bhiman JN, Roark RS, Schramm CA, Gorman J, Chuang GY, Pancera M, Cale EM, Ernandes MJ, Louder MK, et al. New member of the 
V1V2-directed CAP256-VRC26 lineage that shows increased breadth and exceptional potency. J Virol. 2016;90:76-91.

86. Huang J, Kang BH, Ishida E, Zhou T, Griesman T, Sheng Z, Wu F, DoriaRose NA, Zhang B, McKee K, et al. Identification of a CD4-binding-site antibody to HIV that evolved near-pan neutralization breadth. Immunity. 2016;45:1108-21.
Ready to submit your research? Choose BMC and benefit from:

- fast, convenient online submission

- thorough peer review by experienced researchers in your field

- rapid publication on acceptance

- support for research data, including large and complex data types

- gold Open Access which fosters wider collaboration and increased citations

- maximum visibility for your research: over $100 \mathrm{M}$ website views per year

At BMC, research is always in progress.

Learn more biomedcentral.com/submissions 